\title{
Exercise and sports in haemoglobin disorders
}

\author{
D. Yazman \\ Internal Medicıne And Hematology, North Cyprus Turkısh Thalassemia Center, Cyprus
}

\begin{abstract}
Anaemia causes lower oxygen transport to tissues, interfering with normal physical development and may interfere with exercise and sports in thalassemia and Sickle-Cell-Disease (SCD) l. Moreover, in SCD, the abnormal haemoglobin alters the erythrocyte shape and leads to pulmonary parenchymal damage, impaired vascular function and micro vascular complications. However, we now accept that, with regular blood transfusions and efficient oral or parenteral chelation modalities, many patients lead a normal development and life with little or no change from the lifestyle of their unaffected friends, although there are cognitive and emotional factors leading behaviour for participation in the social life and exercise, as studied by many scientists in Italy, United Kingdom, Greece and India.
\end{abstract}

\section{Introduction}

Inherited haemoglobin disorders; the structural haemoglobin variants and the thalassemia, all are caused by defective globin production. Those with a single defective globin gene are carriers or heterozygotes, are symptomless. Those homozygous or compound heterozygotes for the disorder develop symptoms of the disease, mainly anaemia. Anaemia causes lower oxygen transport to tissues, interfering with normal physical development. Evidence based guidelines developed for proper management of thalassemia patients, and the development of new oral iron chelators, all increased expectancy of a long survival of good quality for thalassemia patients. This encourages the patients to plan for their future, having a job, family, children, and other activities like exercise and sports.

Correspondence: D. Yazman, Specialist in Internal Medicıne And Hematology, North Cyprus Turkısh Thalassemıa Center, Cyprus.

Key words: haemoglobin disorders, sports.

(OCopyright D. Yazman., 2011

Licensee PAGEPress, Italy

Thalassemia Reports 2011; 1(s2):e30

doi:10.4081/thal.2011.s2.e30

This article is distributed under the terms of the Creative Commons Attribution Noncommercial License (by-nc 3.0) which permits any noncommercial use, distribution, and reproduction in any medium, provided the original author(s) and source are credited.

Parts of this work were presented at the "12th International Conference on Thalassemia and Hemoglobinopathies”, Antalya (Turkey), 11-14 May 2011.
To overcome this problem, people with thalassemia need adequate blood transfusions to maintain adequate haemoglobin levels (pretransfusion haemoglobin levels need to be kept around 9.5-10 gr/dL) and adequate iron chelation for sparing tissues from the toxic effects of the transfused iron. Some of the consequences of thalassemia influence the person's ability to perform some forms of exercising. Brittle bones which are due to untreated or insufficiently treated anaemia, osteoporosis, or heavy desferrioxamine use will increase the risk of fractures during many types of exercising, but most importantly with heavy weight lifting or resistance training. Anaemia will slow growth and together with iron deposition in endocrine glands will delay puberty, which will result in poor muscle building, short stature, etc. If the person is anaemic, enough oxygen will not be supplied to muscles during exercising resulting in muscle pain and poor muscle and bodybuilding. Heart problems, and restrictive type of lung problems, which are mostly due to iron loading and frequent pulmonary infections resulting from anaemia, are major hindrances against exercising capacity. Sufficiently treated anaemia, will cause iron accumulation in tissues, which when untreated will lead to heart failure and death, accumulation in endocrine organs will cause endocrine abnormalities which again interferes with growth, muscle and bone metabolism, and other endocrine abnormalities (diabetes etc.) Deferoxamine (DFO) toxicity at high doses or at therapeutic doses in patients with hypersensitivity, can be toxic to osteogenesis, collagen synthesis, bone turnover, leading to reduced growth (especially trunk), sliding femoral head, valgus deformity, rachitic like radiological lesions. Bone disease in thalassemia is multifactorial (anaemia, hypogonadism, diabetes, cardiac dysfunction, chronic hepatitis, deferoxamine toxicity, etc), and is already present in childhood. Prevention is the basis of management.

We now accept that all the above statements are valid for only poorly treated or incompliant thalassemia patients whereas in recent era when treatment of the haemoglobin disorders with regular blood transfusions and efficient oral or parenteral chelation modalities is available and we see that many patients lead a normal development and life with little or no change from the lifestyle of their unaffected friends. Exercise itself is a measure to improve blood circulation and tissue oxygenation if enough haemoglobin is present, and hence help growth, muscle and bone building. Smoking cessation, good nutrition with enough protein, calorie, calcium, vitamin D and exercising on a regular basis are important points for maintaining healthy growth and development. Exercise will help prevent fractures and bone deformities. For strong bones, brisk walking, jogging, running, aerobics, step, dancing, circuit training, weight-bearing exercises will be suggested according to the person's medical condition.

There are cognitive and emotional factors affecting behaviour for participation in the social life and exercise, as studied by many scientists in Italy, United Kingdom, Greece and India. Most of them were carried out with interviews using different questionnaires. In 2006, the Indian researchers found that adverse impact of thalassemia was mainly perceived in education (70\%) and sports (72\%). The study population confessed physical weakness as hindering participation in the 
physical activities, but also admitted disappointment for their physical appearances as a negative self concept. ${ }^{1}$

Several studies have conflicting results in thalassemia major patients but most revealed pulmonary functional abnormality presenting as restrictive lung disease with increasing age while the degree and duration of iron overload was explained as the main factor in its pathogenesis. ${ }^{2}$ Dimopoulou ${ }^{3}$ and Piatti ${ }^{4}$ postulating this hypothesis couldn't prove a relationship between restrictive impairment and iron deposition. Later Carnelli et al showed again restrictive lung abnormality as the main lung disorder, but found no clear relation between lung haemosiderosis and restrictive disease. ${ }^{5}$ Post mortem pathological examinations found hemosiderin accumulated in alveolar phagocytes in the perivascular and supporting framework of the lungs ${ }^{6,7}$ However these studies couldn't detect increased fibrous tissue in the lungs. Some groups studied cardiorespiratory function before and 24 hours after transfusion and showed improvement for the group of thalassemia major patients who did not have a history of obstructive lung disease. ${ }^{8}$ This study may suggest the clinician to transfuse the patient 24 hours before the scheduled sports activity, to increase the physical performance.

Due to its different mechanism, sickle cell disease (SCD) has been more prone to research on effects of exercise on the body. In SCD, the abnormal haemoglobin alters the erythrocyte shape and leads to pulmonary parenchymal damage, impaired vascular function and micro vascular complications. Main complications are seen as acute chest syndrome (ACS), reactive airway disease, pulmonary hypertension $(\mathrm{PH})$, and pulmonary fibrosis. $\mathrm{PH}$ is also seen in thalassemics, but while causing mortality in SCD, its impact on the survival of thalassemics is not clear. Sickle cell disease patients should increase their fluid intake and remain warm to prevent red blood cells from sickling associated with hyperviscosity. They are advised to increase consumption of high calorie foods, proteins, and fruits before exercise in order to fight reduced oxygenation of body organs. While vasoocclusive processes are well known for sickle cell disease during uncontrolled exercise, interestingly same pathogenesis has been described for sickle cell trait (carriers) during exercise, and reason described as ampli- fication of adhesion phenomena during exercise, particularly during the late recovery period (24 and 48 hours after the exercise).${ }^{9}$ For thalassemia traits (carriers) no resemblance with thalassemia patients exists with regard to exercise, sports or other properties of the thalassemia disease.

\section{References}

1. Khurana A, Katyal S, Marwaha RK. Psychosocial burden in Thalassemia. Indian J Pediatr 2006;73: 877-880.

2. Factor JM, Pottipati SR, Pappoport I, Rosner IK, Lesser ML, Giardina JP. Pulmonary function abnormalities in thalassemia major and the role of iron overload. Am J Respir Crit Care Med 1994;149:1570-1574.

3. Dimopoulou I, Kremastinos DT, Maris TG, Mavrogeni S, Tzelepis GE. Respiratory function in patients with thalassaemia and iron overload. Eur Respir J 1999;13:602-605.

4. Piatti G, Allegra L, Ambrosetti U, Capellini MD, Turati F, Fiorelli G. B-thalassemia and pulmonary function. Haematologica 1999;84: 804-808

5. Carnelli V, D’Angelo E, Pecchiari M, Ligorio M, D’Angelo E. Pulmonary dysfunction in transfusion dependent patients with thalassemia major. Am J Respir Crit Care Med 2003;168:180-184.

6. Witzleben CL, Wyatt JP. The effect of long term survival on the pathology of thalassemia major. J Path Bact 1961;82:1-12.

7. Cappell DF, Hutchinson HE, Jowett M. Transfusional siderosis: the effects of excessive iron deposits on the tissues. J Path Bact 1957; 74: 245-264.

8. Santamaria F, Villa MP, Werner B, Cutrera R, Barreto M, Ronchetti $\mathrm{R}$. The effect of transfusion on pulmonary function in patients with thalassemia major. Pediatr Pulmonol 1994;18:139-43.

9. Connes P, Hue 0, Tripette J, Hardy-Dessources MD. Blood rheology abnormalities and vascular cell adhesion mechanisms in sickle cell trait carriers during exercise. Clin Hemorheol Microcirc 2008; 39:179-84. 